\title{
Fluctuations and correlations in finite temperature QCD
}

\author{
René Bellwied ${ }^{e}$, Szabolcs Borsányi ${ }^{a}$, Zoltán Fodor ${ }^{a b c}$, Jana Günther ${ }^{a}$, Sándor D. \\ Katz $^{c d}$, Attila Pásztor ${ }^{* a}$, Kálmán K. Szabó ${ }^{a b}$, Claudia Ratti ${ }^{e}$, \\ ${ }^{a}$ University of Wuppertal, Department of Physics, Wuppertal D-42097, Germany \\ ${ }^{b}$ Jülich Supercomputing Center, Jülich D-52425, Germany \\ ${ }^{c}$ Eötvös University, Budapest 1117, Hungary \\ ${ }^{d}$ MTA-ELTE Lendület Lattice Gauge Theory Research Group \\ ${ }^{e}$ University of Houston, Houston, TX 77204, USA
}

\begin{abstract}
We calculate fluctuations and correlations of conserved charges in finite temperature QCD. We also present the equation up to order $\left(\mu_{B} / T\right)^{6}$. This allows reliable calculations of thermodynamic quantities up to $\mu_{B} / T \approx 2$, which covers most of the Beam Energy Scan program at RHIC. Our simulations use staggered quarks with physical quark masses. All of our results are extrapolated to the continuum limit. This conference contribution is mainly based on the papers [1] and [2].
\end{abstract}

38th International Conference on High Energy Physics

3-10 August 2016

Chicago, USA

${ }^{*}$ Speaker. 


\section{QCD in the grand canonical ensemble}

We calculate fluctuations and correlations of conserved charges in finite temperature QCD at zero chemical potential using lattice QCD simulations. These are interesting for their sensitivity to criticality, for probing the composition and distribution of the conserved charges in the QCD medium, and for providing stringent tests on the hadron resonance gas model at low and resummed perturbation theory at high temperatures. They can also be used for extrapolation to small finite chemical potentials. Moreover there is an ongoing effort in the Heavy Ion Experimental community to measure the moments of conserved charge distributions, which can be related one-to-one to fluctuations (with some known caveats). For the fluctuations, or generalized susceptibilities, we use the following notation:

$$
\chi_{i, j, k, l}^{u, d, s, c}=\frac{\partial^{i+j+k+l}\left(p / T^{4}\right)}{\left(\partial \hat{\mu}_{u}\right)^{i}\left(\partial \hat{\mu}_{d}\right)^{j}\left(\partial \hat{\mu}_{s}\right)^{k}\left(\partial \hat{\mu}_{c}\right)^{l}} \quad \chi_{i, j, k}^{B, S, Q}=\frac{\partial^{i+j+k}\left(p / T^{4}\right)}{\left(\partial \hat{\mu}_{B}\right)^{i}\left(\partial \hat{\mu}_{S}\right)^{j}\left(\partial \hat{\mu}_{Q}\right)^{k}}
$$

where $\hat{\mu}=\mu / T$. The relationship between the chemical potentials:

$$
\mu_{u}=\frac{1}{3} \mu_{B}+\frac{2}{3} \mu_{Q} \quad \mu_{d}=\frac{1}{3} \mu_{B}-\frac{1}{3} \mu_{Q} \quad \mu_{s}=\frac{1}{3} \mu_{B}-\frac{1}{3} \mu_{Q}-\mu_{S}
$$

allows one to convert the two types of derivatives into each other and write say the $\chi_{i, j, k}^{B, S, Q}$ as linear combinations of the $\chi_{i, j, k}^{u, d, s}$.These fluctuations then can be used to extrapolate the equation of states to small but general values of $\mu_{B}, \mu_{Q}, \mu_{S}$, where we have:

$$
\frac{p}{T^{4}}=\sum_{i, j, k} \frac{1}{i ! j ! k !} \chi_{i j k}^{B S Q}(T) \hat{\mu}_{B}^{i} \hat{\mu}_{S}^{j} \hat{\mu}_{Q}^{k}
$$

If we restrict outselves to conditions present in Heavy Ion Collisions, namely $\left\langle n_{S}\right\rangle=0$ and $\left\langle n_{Q}\right\rangle=$ $0.4\left\langle n_{B}\right\rangle$, we get an expansion of the form:

$$
\frac{p}{T^{4}}=c_{0}(T)+c_{2}(T) \cdot \hat{\mu}_{B}^{2}+c_{4}(T) \cdot \hat{\mu}_{B}^{4}+c_{6}(T) \cdot \hat{\mu}_{B}^{6}+\ldots
$$

This gives the finite chemical potential equation of state relevant to hydrodynamic simulations of heavy ion collisions. We will also present results on these coefficients. The equation of state of QCD at $\mu_{B}=0$ is known for a while $[3,4,5]$, and is a standard component of hydrodynamic description of heavy ion collisions. The corrections at finite density are much harder to calculate $[6,7]$. Our work is an important step in the finite $\mu$ direction.

\section{The two methods to calculate fluctuations}

We will use two distinct method to calculate the fluctuations. First, we can calculate the $\mu$ derivatives directly at zero chemical potentials $[8,9]$. This has the advantage that there is no additional systematic error coming from fitting procedure. However, higher derivatives are very noisy. This traditional technique finds the $c_{4}$ and $c_{6}$ coefficients from the non-Gaussianity of the fluctuations of conserved charges. In large simulation volumes, however, the central limit theorem reduces these below the level of detection. With physical quark masses, fine and large lattices one must seek for an alternative technique. 
The method we use is to simulate at imaginary chemical potential[10,11,12,13,14], do a fit for the $\mu$ dependence of different observables, and deduce the derivatives at 0 that way. This makes higher accuracy possible with the same amount of computer time, however it has systematic errors coming from fitting, as at imaginary $\mu$ you have an exact result, containing all orders of the Taylor expansion. This is illustrated on Figure 1. Such systematic errors are included in all of our result plots.

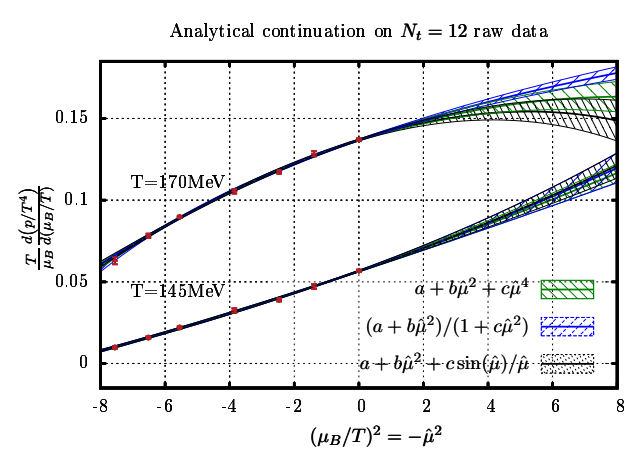

Figure 1: Illustration of the systematic error coming from analytic continuation with different fit ansatzes. The systematic error coming from the choice of these 3 ansatzes is included in out results.

\section{Lattice details}

The susceptibilities can be expressed as fermionic matrix traces, that can be evaluated on the lattice by utilizing a large number of Gaussian random sources. Our continuum extrapolation for the imaginary chemical potential data is based on the following lattices: $40^{3} \times 10,48^{3} \times 12$ and $64^{3} \times 16$, in the 4 stout staggered discretization. For the details of the lattice action and ensembles see Ref. [1, 15]. For the zero chemical potential data in the cross-over region we use aspect ratios between 3 and 4 and temporal extent $N_{t}=8 \ldots 24$. For temperatures $T \geq 300 \mathrm{MeV}$ we do not keep the lattice geometry constant in our temperature scan, but keep the physical volume constant, with $L T_{c}>2$. So e.g. for $N_{t}=16$ we have the lattices:

\begin{tabular}{|c|c|c|c|c|}
\hline $64^{3} \times 16$ & $80^{3} \times 16$ & $96^{3} \times 16$ & $112^{3} \times 16$ & $128^{3} \times 16$ \\
\hline $300 \mathrm{MeV}$ & $360 \mathrm{MeV}$ & $440 \mathrm{MeV}$ & $520 \mathrm{MeV}$ & $600 \mathrm{MeV}$ \\
\hline
\end{tabular}

Keeping the volume large is important for testing perturation theory.

For some observables, like $\chi_{2}^{B}$, there is a long range of safe linear extrapolation, but observables that are related to pion physics, like $\chi_{2}^{Q}$, show a strong non-linear $1 / N_{t}$ dependence, and only for very fine lattices, $N_{t} \geq 16$ we see a linear regime. No such problem appears for the observables considered in our imaginary chemical potential runs.

For the imaginary $\mu$ runs, the tuning of the chemical potentials such that $\left\langle n_{S}\right\rangle=0$ and $\left\langle n_{Q}\right\rangle=$ $0.4\left\langle n_{B}\right\rangle$ are satisfied is an additional issue. The method used for tuning the strangeness neutrality condition was first introduced in [16]. 


\section{Results}

On Figure 2 and Figure 3 (left) we show several generalized susceptibilities in finite temperature QCD together with some model predictions. All the observables we consider show an agreement with the HRG model up to $T \sim 150-155 \mathrm{MeV}$ The diagonal second derivatives start to agree with the perturbative results $[17,18]$ earlier than the off-diagonal derivatives. In the high temperature range a simular calculation to ours was also presented in [19].

In addition to the HRG we show a naive quasiparticle estimate for the charm susceptibility. The mass of the charm quark was fitted to the last points $\left(m_{c}^{Q P}=1430 \mathrm{MeV}\right)$. The charm quark mass $\left(m_{c}^{Q P}=1430 \mathrm{MeV}\right)$ is empirical, and may depend on the range of the matching to the data. The quasiparticle model's results are overestimating the lattice data below approx. 350MeV. This leaves room for multiple interpretations, like a $T$-dependent $m_{c}^{Q P}$, limitations of the quasiparticle model or bound states absorbing free quarks.

The coefficients $c_{n}$ of the expansion of the pressure in $\mu_{B}$, satisfying $\left\langle n_{S}\right\rangle=0$ and $\left\langle n_{Q}\right\rangle=$ $0.4\left\langle n_{B}\right\rangle$ cen be seen on the left side of Figure 3 .

In ideal hydrodynamics, we have $S / N=$ fixed. These trajectories can be readily calculated from the equation of state given by the $c_{n}$ coefficients, once the value of $S / N$ is fixed. To fix these value for the value relevant for the RHIC beam energy scan we used the freeze-out temperature and chemical potential estimates from Ref. [20]. The resulting trajectories can be seen in the left hand side of Figure 4. The equation of state along these trajectories is on the right side of Figure 4. The equation of state can be reliably calculated for $\hat{\mu}_{B} \leq 2$, or in terms of the RHIC energy scan, for the center of mass energies:

$$
\sqrt{s}=200,62.4,39,27,19.6,14.5 \mathrm{GeV}
$$

For the use of $\mu_{S}$ and a function of $\mu_{B}$ and $T$, determined from our simulations, to Kaon freeze-out, see Ref. [21].

\section{Acknowledgment}

This project was funded by the DFG grant SFB/TR55. This material is based upon work supported by the National Science Foundation through grant number NSF PHY-1513864 and by the U.S. Department of Energy, Office of Science, Office of Nuclear Physics, within the framework of the Beam Energy Scan Theory (BEST) Topical Collaboration. An award of computer time was provided by the INCITE program. This research used resources of the Argonne Leadership Computing Facility, which is a DOE Office of Science User Facility supported under Contract DE-AC02-06CH11357. The authors gratefully acknowledge the Gauss Centre for Supercomputing (GCS) for providing computing time for a GCS Large-Scale Project on the GCS share of the supercomputer JUQUEEN at the Jülich Supercomputing Centre.

\section{References}

[1] R. Bellwied, S. Borsanyi, Z. Fodor, S. D. Katz, A. Pasztor, C. Ratti and K. K. Szabo, Phys. Rev. D 92 (2015) no.11, 114505 doi:10.1103/PhysRevD.92.114505 [arXiv:1507.04627 [hep-lat]]. 

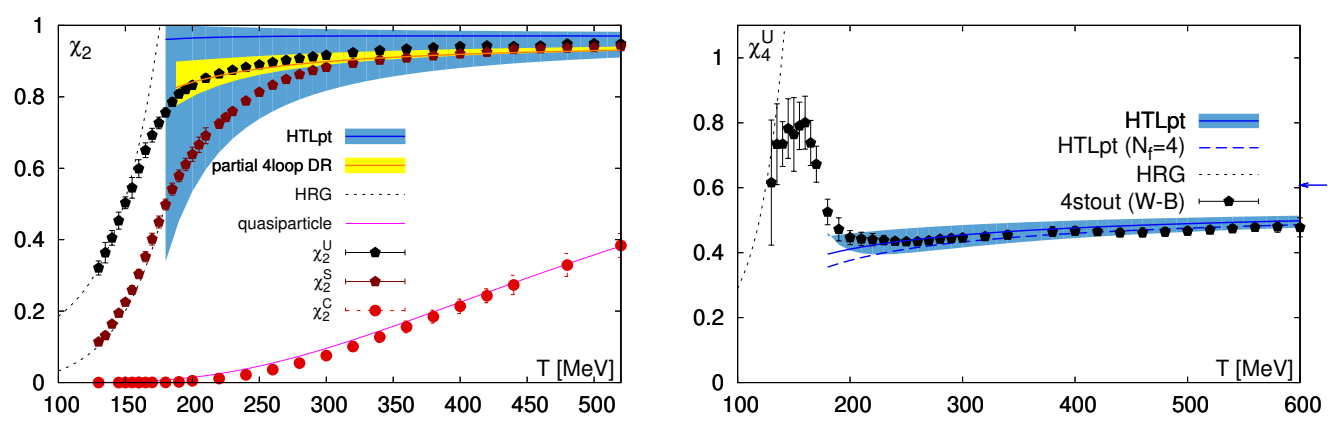

Figure 2: Second order susceptibilities (left) and fourth order diagonal susceptibilities(right). Various model estimates are also shown.
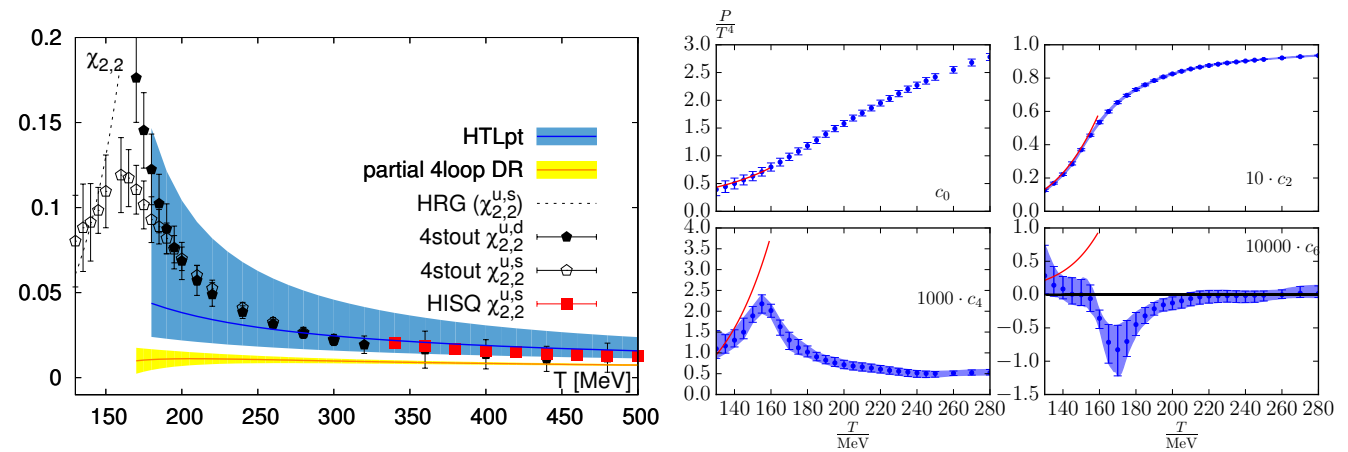

Figure 3: Forth order diagonal susceptibilities(left) and the coefficients of the equation of state with the constraints $\left\langle n_{S}\right\rangle=0$ and $\left\langle n_{Q}\right\rangle=0.4\left\langle n_{B}\right\rangle$ (right). The hadron resonance gas model predictions are shown in red, for comparison.
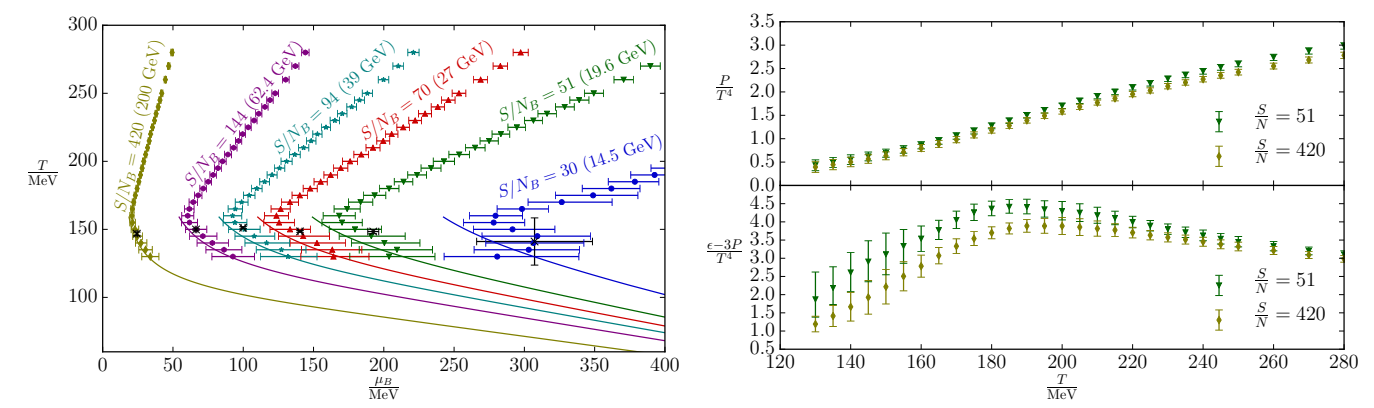

Figure 4: The isentropic trajectories(left) and the equation of state along these isentropic trajectories(right).

[2] J. Gunther, R. Bellwied, S. Borsanyi, Z. Fodor, S. D. Katz, A. Pasztor and C. Ratti, arXiv:1607.02493 [hep-lat].

[3] S. Borsanyi, G. Endrodi, Z. Fodor, A. Jakovac, S. D. Katz, S. Krieg, C. Ratti and K. K. Szabo, JHEP 1011, 077 (2010) doi:10.1007/JHEP11(2010)077 [arXiv:1007.2580 [hep-lat]]. 
[4] S. Borsanyi, Z. Fodor, C. Hoelbling, S. D. Katz, S. Krieg and K. K. Szabo, Phys. Lett. B 730, 99 (2014) doi:10.1016/j.physletb.2014.01.007 [arXiv:1309.5258 [hep-lat]].

[5] A. Bazavov et al. [HotQCD Collaboration], Phys. Rev. D 90, 094503 (2014) doi:10.1103/PhysRevD.90.094503 [arXiv:1407.6387 [hep-lat]].

[6] S. Borsanyi, G. Endrodi, Z. Fodor, S. D. Katz, S. Krieg, C. Ratti and K. K. Szabo, JHEP 1208, 053 (2012) doi:10.1007/JHEP08(2012)053 [arXiv:1204.6710 [hep-lat]].

[7] P. Hegde [BNL-Bielefeld-CCNU Collaboration], PoS LATTICE 2014, 226 (2014) [arXiv:1412.6727 [hep-lat]].

[8] C. R. Allton, S. Ejiri, S. J. Hands, O. Kaczmarek, F. Karsch, E. Laermann, C. Schmidt and L. Scorzato, Phys. Rev. D 66, 074507 (2002) doi:10.1103/PhysRevD.66.074507 [hep-lat/0204010].

[9] O. Kaczmarek et al., Phys. Rev. D 83, 014504 (2011) doi:10.1103/PhysRevD.83.014504 [arXiv:1011.3130 [hep-lat]].

[10] P. de Forcrand and O. Philipsen, Nucl. Phys. B 642, 290 (2002) doi:10.1016/S0550-3213(02)00626-0 [hep-lat/0205016].

[11] M. D’Elia and M. P. Lombardo, Phys. Rev. D 67, 014505 (2003) doi:10.1103/PhysRevD.67.014505 [hep-lat/0209146].

[12] M. D’Elia, F. Di Renzo and M. P. Lombardo, Phys. Rev. D 76, 114509 (2007) doi:10.1103/PhysRevD.76.114509 [arXiv:0705.3814 [hep-lat]].

[13] P. de Forcrand and O. Philipsen, JHEP 0811, 012 (2008) doi:10.1088/1126-6708/2008/11/012 [arXiv:0808.1096 [hep-lat]].

[14] M. D’Elia and F. Sanfilippo, Phys. Rev. D 80, 014502 (2009) doi:10.1103/PhysRevD.80.014502 [arXiv:0904.1400 [hep-lat]].

[15] S. Borsanyi et al., Nature 539, no. 7627, 69 (2016) doi:10.1038/nature20115 [arXiv:1606.07494 [hep-lat]].

[16] R. Bellwied, S. Borsanyi, Z. Fodor, J. Günther, S. D. Katz, C. Ratti and K. K. Szabo, Phys. Lett. B 751, 559 (2015) doi:10.1016/j.physletb.2015.11.011 [arXiv:1507.07510 [hep-lat]].

[17] N. Haque, A. Bandyopadhyay, J. O. Andersen, M. G. Mustafa, M. Strickland and N. Su, JHEP 1405, 027 (2014) doi:10.1007/JHEP05(2014)027 [arXiv:1402.6907 [hep-ph]].

[18] S. Mogliacci, J. O. Andersen, M. Strickland, N. Su and A. Vuorinen, JHEP 1312, 055 (2013) doi:10.1007/JHEP12(2013)055 [arXiv:1307.8098 [hep-ph]].

[19] H.-T. Ding, S. Mukherjee, H. Ohno, P. Petreczky and H.-P. Schadler, Phys. Rev. D 92, no. 7, 074043 (2015) doi:10.1103/PhysRevD.92.074043 [arXiv:1507.06637 [hep-lat]].

[20] P. Alba, W. Alberico, R. Bellwied, M. Bluhm, V. Mantovani Sarti, M. Nahrgang and C. Ratti, Phys. Lett. B 738, 305 (2014) doi:10.1016/j.physletb.2014.09.052 [arXiv:1403.4903 [hep-ph]].

[21] J. Noronha-Hostler, R. Bellwied, J. Gunther, P. Parotto, A. Pasztor, I. P. Vazquez and C. Ratti, arXiv:1607.02527 [hep-ph]. 